\title{
Plan national santé-environnement 2004-2008
}

\author{
Franchir une nouvelle étape dans la prévention des conséquences sanitaires des atteintes de l'environnement
}

Ministère de la santé et de la protection sociale, Ministère de l'écologie et du développement durable, Ministère de l'emploi, du travail et de la cohésion sociale, Ministère délégué à la recherche

Les impacts de l'environnement sur notre santé sont manifestes. Parmi les constats récents peuvent être relevés les éléments suivants, qui doivent être perçus comme un véritable signal d'alarme:

- de l'ordre de 30000 décès anticipés par an sont liés à la pollution atmosphérique urbaine;

- un doublement de la prévalence des maladies allergiques respiratoires est enregistré depuis 20 ans;

- seules $37 \%$ des ressources en eau potable disposent aujourd'hui de périmètres de protection;

- la qualité de l'eau dans le milieu naturel se dégrade de manière continue;

- $14 \%$ des couples consultent pour des difficultés à concevoir, lesquelles difficultés pourraient être liées à des expositions à des substances toxiques pour la reproduction;

- 7 à $20 \%$ des cancers seraient imputables à des facteurs environnementaux;

- près d'un million de travailleurs seraient exposés à des substances cancérogènes;

- l'évaluation des risques liés aux substances chimiques est insuffisante, les capacités d'expertise française trop peu développées;

- les risques liés aux expositions dans les locaux sont mal connus alors que la population y séjourne environ $80 \%$ de son temps;

- la recherche, l'expertise, la formation et l'information en matière de santé environnement sont très insuffisamment développées.

Face à un tel constat, il y a urgence à agir, pour empêcher que ces impacts aillent à l'encontre de l'amélioration de l'espérance de vie. Le Gouvernement français a décidé de s'attaquer résolument au problème, en élaborant un Plan National Santé Environnement. Ce plan a pour objectif de rendre notre environnement plus respectueux de notre santé en limitant les polluants et risques qu'il véhicule. Malgré les efforts importants engagés pour réduire les rejets de polluants, pour renforcer les moyens de l'expertise en appui à la décision, ainsi que pour prévenir les principaux risques sanitaires environnementaux, une étape nouvelle dans la lutte contre les risques sanitaires liés à l'environnement est indispen- sable, dans une perspective de développement durable. Celle-ci doit prendre en compte l'ensemble des polluants et des milieux de vie.

Cette démarche s'inscrit dans un contexte européen et international marqué par une mobilisation grandissante. En témoignent la $4^{\mathrm{e}}$ conférence ministérielle sur l'environnement et la santé organisée à l'initiative de la région Europe de l'OMS du 23 au 25 juin à Budapest en 2004, les progrès accomplis depuis le lancement de ce cycle de conférences, ainsi que la stratégie et le plan d'action communautaire SCALE [1] élaborés à cette occasion.

La construction du PNSE s'appuie sur le rapport d'une «Commission d'orientation» composée d'experts. Ce rapport, remis au Premier Ministre le 12 février 2004, établit un diagnostic de l'exposition des français aux pollutions environnementales dans leur vie quotidienne et recommande des priorités d'actions. Tous les milieux de vie sont abordés pour la première fois de façon conjointe: environnement domestique, extérieur, mais aussi environnement professionnel.

Cette construction s'est également nourrie des observations formulées au cours du processus de consultation d'instances consultatives spécialisées et de la société civile.

Le PNSE est un premier plan qui définit les actions qui structureront l'action du gouvernement au cours des cinq années à venir. D'autres plans quinquennaux suivront. Un bilan à miparcours sera réalisé en vue de mesurer les progrès accomplis et de décider des éventuelles améliorations à apporter.

Le PNSE comporte quarante-cinq actions, dont douze ont été identifiées comme prioritaires. L'ensemble vise à répondre à 3 objectifs majeurs:

- respirer un air et boire une eau de bonne qualité;

- prévenir les pathologies d'origine environnementale et notamment les cancers;

- mieux informer le public et protéger les populations sensibles (enfants et femmes enceintes).

Les douze actions prioritaires sont les suivantes:
Legislation Evaluation = Science Enfants Vigilance Législation Evaluation. 


\section{Garantir un air et une eau de bonne qualité}

1.1 Réduire les émissions de particules diesel par les sources mobiles

Les particules fines, et en particulier celles émises par les véhicules diesels, constituent l'un des principaux facteurs de risque sanitaire lié à la pollution atmosphérique en milieu urbain. L'adoption pour 2010 de normes d'émission européennes exigeantes pour les véhicules particuliers sera recherchée. Au plan national, des dispositifs d'incitations à l'achat de véhicules légers équipés de technologies permettant une quasi suppression des émissions de particules fines et à l'équipement des poids lourds existants seront mis en place.

\subsection{Réduire les émissions aériennes} de substances toxiques d'origine industrielle Certaines substances émises ou utilisées par des installations classées sont susceptibles d'affecter la santé des populations en raison de leur caractère dangereux. De nouveaux objectifs sont fixés à horizon 2010 afin d'obtenir une diminution des émissions dans l'air de $85 \%$ pour les dioxines, $50 \%$ pour le cadmium, $65 \%$ pour le plomb, $40 \%$ pour le chlorure de vinyle monomère et de $35 \%$ pour le benzène (années de référence 2000 ou 2002 selon les cas).

\subsection{Assurer une protection de la totalité des captages d'eau potable}

La simplification des procédures de mise en place des périmètres de protection des captages, le renforcement des moyens de contrôle et d'instruction des DDASS et l'incitation des collectivités locales permettront de protéger 100\% des captages d'ici 2010. Ces mesures, ainsi que la fiabilisation des systèmes, viseront à diminuer par 2 d'ici 2008 le nombre d'habitants desservis par une eau non conforme.

\subsection{Mieux connaître les déterminants de la qualité de l'air intérieur}

Le renforcement et l'extension des activités menées par l'observatoire de la qualité de l'air intérieur (OQAI) permettront d'évaluer l'exposition de la population aux polluants de l'air dans différents locaux. Les principales sources de pollution seront identifiées afin d'élaborer à miparcours du PNSE les premières mesures de prévention et de réduction des risques sanitaires.
1.5 Mettre en place un étiquetage des caractéristiques sanitaires et environnementales des matériaux de construction

Une méthodologie globale d'évaluation des impacts sanitaires et environnementaux des émissions sera précisée et appliquée, dans un premier temps, aux émissions de composés organiques volatils (COV) et de formaldéhyde. Une démarche volontaire d'étiquetage et d'affichage des performances sanitaires et environnementales sera proposée. $50 \%$ des produits et matériaux nouvellement mis sur le marché en 2008 devront avoir fait l'objet d'un examen et de l'étiquetage correspondant.

\section{Prévenir les pathologies d'origine environnementale et notamment les cancers}

\subsection{Renforcer les capacités d'évaluation des risques sanitaires des substances chimiques dangereuses}

Afin de mieux évaluer les risques sanitaires présentés par plusieurs familles de substances dont certains de leurs composants font déjà l'objet de préoccupations: éthers de glycol, fibres minérales artificielles, pesticides, phtalates, hydrocarbures, etc. les capacités d'expertise en France seront renforcées. Ce renforcement portera prioritairement sur les risques en milieu professionnel où l'Etat entend renforcer notamment ses capacités de veille aux expositions des travailleurs aux substances chimiques ainsi que sur les pesticides, compte tenu de leurs caractères intrinsèques de dangerosité et de persistance.

\subsection{Réduire les expositions professionnelles} aux agents cancérogènes, mutagènes et reprotoxiques

Dès 2005 l'application de la réglementation visant à réduire les expositions en milieu du travail aux agents CMR sera accélérée en ce qui concerne les poussières de bois, le benzène, le plomb et les fibres céramiques réfractaires: utilisation de méthodes de mesures innovantes, diffusion de guide de bonnes pratiques, abaissement des valeurs limites d'exposition, modernisation et renforcement des moyens de contrôles de l'inspection du travail. Parallèlement des pratiques innovantes pour la prévention des risques à long terme seront expérimentées dans les services de santé du travail. 
2.3 Renforcer les connaissances fondamentales des déterminants environnementaux et sociétaux de la santé des populations et le développement de nouvelles méthodes en sciences expérimentales

Un programme de recherche inter-organismes pour l'amélioration des connaissances scientifiques sera mis en œuvre. Il permettra d'améliorer la compréhension de l'impact des différents facteurs environnementaux sur la santé humaine et sur l'apparition des pathologies (maladies respiratoires, cardio-vasculaires, neurologiques, inflammatoires, immunologiques et métaboliques, etc.). Il permettra également d'approfondir la connaissance et la modélisation de l'évolution des substances toxiques, de leur spéciation et de leurs transformations (produits en traces, en mélange et en interaction) au sein des différents compartiments de l'environnement et de mettre au point de nouveaux outils de mesure. Les différents milieux (atmosphère, écosystèmes aquatiques et terrestres, sols et sous-sols, anthroposystèmes, lieux de travail et de vie) et vecteurs (aliments) seront pris en compte.

\section{Mieux informer le public et protéger les populations sensibles}

\subsection{Faciliter l'accès à l'information en santé-} environnement et favoriser le débat public Pour améliorer l'information du public et des professionnels sur les risques sanitaires d'origine environnementale, sur les moyens de s'en prémunir et sur les mesures et les politiques qui sont mises en œuvre, l'AFSSE créera un site portail sur Internet spécifiquement dédié. Ce site constituera la référence au plan national, donnant accès à une information complète et actualisée incluant les principales sources scientifiques et techniques. Une attention particulière sera portée à l'information des professionnels en contact avec les populations sensibles (enfants, adolescents, personnes âgées, patients atteints d'une affection de longue durée) ou susceptibles de détecter des situations spécifiques (zones particulièrement polluées, situation de travail, habitat dégradé).

3.2 Améliorer la prévention du saturnisme infantile, le dépistage et la prise en charge des enfants intoxiqués

Une enquête nationale de prévalence du saturnisme infantile sera lancée ainsi que des pro- grammes ciblés de dépistage dans les zones prioritaires; l'exonération du ticket modérateur de la plombémie qui sera mise en œuvre facilitera le dépistage. Parallèlement, les mesures incitatives ou coercitives visant à faire réaliser les travaux nécessaires dans les bâtiments prévus par la loi de santé publique seront mises en œuvre et appuyées par l'action des DDASS dont les moyens seront renforcés. Enfin une action sera menée à proximité des sites industriels émetteurs de plomb (actuels et passés): recensement et en tant que de besoin, campagnes de mesures, évaluation des risques détaillées.

\subsection{Réaliser une étude épidémiologique sur les enfants}

A l'instar des travaux similaires entamés aux Etats-Unis et en collaboration avec divers pays européens, ce projet vise à mettre en place une étude épidémiologique dite «de cohorte» regroupant 20000 enfants qui seront suivis de la période gestationnelle jusqu'à l'âge adulte. Les pathologies susceptibles d'être liées à l'environnement (notamment les cancers, les troubles reprotoxiques et neurotoxiques) et les expositions (y compris pré-conceptionnelles ou in utero) aux principaux polluants seront surveillées chez ces enfants. L'étude des relations pathologies/expositions pourra conduire à la réduction des émissions de certains polluants ou à l'interdiction de certaines substances chimiques.

\subsection{Réduire I'incidence de la légionellose}

Un programme d'actions spécifiques est mis en place afin de réduire de $50 \%$ l'incidence de la légionellose qui touche principalement des populations fragilisées (silicose, pathologies respiratoires). Il prévoit notamment l'amélioration des connaissances, un renforcement de la réglementation sur la conception et le fonctionnement des tours aéro-réfrigérantes, le recensement de ces tours, ainsi que des mesures de prévention liées aux réseaux d'eau chaude sanitaire.

\section{Annexe}

Le plan contient au total 45 actions, organisées en 8 axes qui recouvrent l'ensemble du champ des relations entre environnement et santé. Un rapport complet, accessible sur les sites Internet des Ministères chargés de la santé et de l'environnement, détaille le contenu de l'ensemble de ces mesures dont voici la liste: 


\section{Prévenir les décès liés aux infections/ intoxications aiguës}

\section{Constat}

Dans certaines situations, l'environnement peut avoir pour conséquence des décès brutaux. C'est le cas des infections par la légionellose, des intoxications par le monoxyde de carbone ou de certaines situations climatiques extrêmes. Des mesures de prévention efficaces sont à mettre en œuvre.

\section{Actions}

- Action 1 (prioritaire): Réduire de $50 \%$ l'incidence de la légionellose à l'horizon 2008.

- Action 2: Réduire de 30\% la mortalité par intoxication au monoxyde de carbone à l'horizon 2008.

- Action 3: Maîtriser les risques sanitaires liés aux températures extrêmes.

\section{Protéger la santé en améliorant la qualité des milieux (air et eau)}

\section{Constat}

De nombreuses études épidémiologiques montrent que la pollution atmosphérique, notamment en milieu urbain, constitue l'un des facteurs principaux de risques sanitaires (cancers, insuffisances respiratoires, maladies cardio-vasculaires, asthmes) liés à l'environnement [2]. La dégradation de la qualité des ressources en eau $\mathrm{du}$ fait de pollutions ponctuelles ou diffuses entraîne d'une part une obligation de mettre en œuvre des traitements coûteux pour la rendre potable [3] et, d'autre part, une augmentation de risques sanitaires par contact direct (p.ex. baignade).

\section{Actions}

\subsection{Air et Transports}

- Action 4 (prioritaire): Réduire les émissions de particules diesel par les sources mobiles.

- Action 5: Promouvoir les modes de déplacements alternatifs.

- Action 6: Mieux prendre en compte l'impact sur la santé des projets d'infrastructure de transports.

\subsection{Air et sources fixes}

- Action 7 (prioritaire): Réduire les émissions aériennes de substances toxiques d'origine industrielle.

- Action 8: Réduire les émissions de $\mathrm{NO}_{\mathrm{X}}$ des installations industrielles.
- Action 9: Réduire les émissions polluantes du secteur résidentiel tertiaire.

2.3 Préservation des ressources en eaux et des sols

- Action 10 (prioritaire): Améliorer la qualité de l'eau potable en préservant les captages des pollutions ponctuelles et diffuses.

- Action 11: Limiter les pollutions des eaux et des sols dues aux pesticides et à certaines substances potentiellement dangereuses.

- Action 12: Prévenir et réduire les risques spécifiques d'exposition au mercure en Guyane et aux pesticides en Guadeloupe et Martinique.

- Action 13: Diminuer le risque sanitaire dû à la baignade.

\section{Protéger la population à l'intérieur des locaux}

\section{Constat}

Nous passons de 80 à $90 \%$ de notre temps dans des lieux clos: habitations, lieux de travail, moyens de transport, écoles, dans lesquels nous respirons un air différent de l'air extérieur. A la pollution provenant de l'extérieur s'ajoutent des polluants issus de trois principales sources: les appareils à combustion (monoxyde de carbone, dioxyde d'azote), les constituants du bâtiment, incluant les équipements et mobiliers (plomb des peintures, formaldéhyde, composés organiques volatils, fibres de toutes sortes) et l'activité humaine (produits ménagers, bricolage, acariens, moisissures, etc.).

\section{Actions}

- Action 14 (prioritaire): Mieux connaître les déterminants de la qualité de l'air intérieur et renforcer la réglementation.

- Action 15 (prioritaire): Mettre en place un étiquetage des caractéristiques sanitaires et environnementales des matériaux de construction.

- Action 16: Améliorer l'information des acquéreurs et des futurs locataires de biens immobiliers sur leurs principales caractéristiques techniques.

- Action 17: Réduire l'exposition au radon dans les bâtiments à usage d'habitation et mieux évaluer le risque.

- Action 18: Limiter l'exposition de la population aux fibres minérales artificielles.

- Action 19: Protéger la santé des populations vivant en habitat insalubre. 


\section{Mieux maîtriser les risques liés aux substances chimiques}

\section{Constat}

Parmi 100000 substances chimiques [4], seules quelques milliers ont déjà fait l'objet d'une évaluation approfondie des risques sanitaires qu'elles sont susceptibles de présenter.

Cette incertitude s'avère préoccupante, en particulier quant aux effets à long terme des expositions à faibles doses, en milieu professionnel comme en population générale: développement de pathologies comme les cancers ou les troubles du développement, allergies.

\section{Actions}

- Action 20 (prioritaire): Renforcer les capacités d'évaluation des risques sanitaires liés aux substances chimiques.

- Action 21: Développer des outils pour mieux évaluer les risques sanitaires des substances chimiques ou biologiques.

- Action 22: Renforcer la surveillance du marché notamment par la réalisation de campagnes ciblées de contrôle.

- Action 23 (prioritaire): Réduire les expositions professionnelles aux agents cancérogènes, mutagènes et reprotoxiques (CMR), notamment celles concernant les poussières de bois, le benzène, le plomb et les fibres céramiques réfractaires, en renforçant et en modernisant les moyens de contrôle et les services de santé et sécurité au travail.

\section{Renforcer la protection des enfants et des femmes enceintes}

\section{Constat}

Il est essentiel de porter une attention spéciale aux enfants car ils présentent une vulnérabilité particulière aux agressions par des agents environnementaux. Il y a à cela des raisons biologiques et physiologiques. Ainsi, leur capacité d'élimination et de lutte contre les effets des substances toxiques est moindre que celle des adultes. Les polluants peuvent modifier ou endommager de façon irréversible les organes en développement.

\section{Actions}

- Action 24: Renforcer, notamment en milieu professionnel, la protection, des femmes enceintes et la préservation de la fertilité masculine.

4 Dont 30000 sont mises sur le marché en quantité supérieure à une tonne par an en Europe.
- Action 26 (prioritaire): Réaliser une étude épidémiologique enfants en lien avec l'étude américaine (National Children Study).

- Action 27: Améliorer l'information sur la prévention de l'asthme et des allergies.

- Action 28: Protéger les adolescents des risques dus à la musique amplifiée.

- Action 29: Veiller à la qualité des bâtiments accueillant des enfants.

\section{Mobiliser et développer le potentiel de recherche}

\section{Constat}

L'appréhension de l'influence des facteurs environnementaux sur la santé est un vaste enjeu scientifique: il s'agit de lier la compréhension de l'apparition des pathologies aux conditions environnementales et d'exposition, mais aussi de comprendre les facteurs qui concourent à la protection de la santé des populations et les conditions qui doivent être réunies pour qu'une action en faveur de la santé ait les effets attendus. Ainsi la recherche scientifique a un rôle majeur à jouer pour mesurer, diagnostiquer les phénomènes et comprendre leur dynamique d'évolution.

\section{Actions}

- Action 30: Renforcer la coordination de la recherche dans le domaine santé environnement.

- Action 31: Soutenir la création d'un grand programme scientifique international et renforcer la participation de la recherche française dans les programmes européens et internationaux.

- Action 32: Former des jeunes chercheurs et enseignants chercheurs en santé environnement et développer le potentiel humain.

- Action 33 (prioritaire): Actions de soutien à la recherche sur des thèmes stratégiques.

- Action 34: Renforcer et coordonner les appels à propositions de recherche en appui aux politiques publiques.

\section{Améliorer les dispositifs de veille, de surveillance et d'alerte}

\section{Constat}

Les systèmes d'information en santé environnementale visent d'une part, à documenter et, d'autre part, à surveiller les émissions de polluants et leur impact sur la qualité des milieux, ainsi que l'exposition de la population à ces nuisances au travers de ses différents vecteurs et leurs conséquences sanitaires. Le constat est que 
les systèmes d'information actuels répondent à des besoins distincts et sont par conséquent insuffisamment exploités dans cet esprit. Un effort particulier doit être consacré aux dispositifs d'alerte, qui reposent en partie sur les systèmes d'information, et à la veille afin de garantir la prise en compte suffisamment en amont des risques émergents ou des nouvelles données scientifiques.

\section{Actions}

- Action 35: Améliorer la performance et l'intégration des systèmes d'information en santé environnement.

- Action 36: Organiser l'exploitation des données existantes pour estimer l'exposition de la population aux pesticides.

- Action 37: Etudier les modalités d'utilisation des indicateurs biologiques d'exposition en milieu professionnel et en population générale.

- Action 38: Mieux connaître la santé des travailleurs et les expositions professionnelles pour réduire le nombre de maladies d'origine professionnelle.

- Action 39: Développer les systèmes d'alerte et renforcer le réseau national de toxico-vigilance.

- Action 40: Animer un réseau de veille en santé-environnement en appui aux politiques de prévention et précaution.

\section{Consolider la formation et développer I'information}

\section{Constat}

Il n'existe pas de véritable culture de l'environnement et de ses relations avec la santé, qui soit suffisamment diffusée au sein de la population pour permettre une implication, pourtant essentielle, du citoyen dans la prise de décision publique et pour influencer notablement les comportements individuels et collectifs. Les professionnels des différents secteurs (médecins, personnel médical et para médical, administrations, industriels, etc.) sont encore peu formés et mobilisés sur ce type d'approche transversale.

\section{Actions}

- Action 41: Intégrer la dimension santé-environnement dans les formations initiales.

- Action 42: Intégrer la dimension santé-environnement dans la formation continue des professionnels de santé.

- Action 43: Développer l'information et la formation des différents acteurs de la prévention dans l'entreprise.

- Action 44 (prioritaire): Faciliter l'accès à l'information en santé-environnement et favoriser le débat public.

- Action 45: Consacrer la fête de la science en 2006 au thème santé-environnement. 